\title{
Re-Irradiation for Recurrent Glioblastoma Multiforme
}

\author{
DIRK RADES ${ }^{1}$, JASPAR WITTELER $^{1}$, JAN LEPPERT $^{2}$ and STEVEN E. SCHILD ${ }^{3}$ \\ ${ }^{1}$ Department of Radiation Oncology, University of Lübeck, Lübeck, Germany; \\ ${ }^{2}$ Department of Neurosurgery, University of Lübeck, Lübeck, Germany; \\ ${ }^{3}$ Department of Radiation Oncology, Mayo Clinic, Scottsdale, AZ, U.S.A.
}

\begin{abstract}
Background/Aim: Patients requiring re-irradiation for recurrent glioblastoma multiforme (GBM) may benefit from individualized therapy. This study aimed to identify predictors of survival and contribute to treatment personalization. Patients and Methods: In 28 patients with recurrent GBM, nine factors were analyzed for associations with survival: Main location and type of recurrence, Karnofsky performance score (KPS), age, gender, interval between primary radiotherapy and recurrence, gross total resection (GTR), equivalent dose in 2-Gy fractions (EQD2) of re-irradiation and cumulative EQD2 of primary and re-irradiation. Results: On univariate analyses, GTR ( $p=0.047)$, $E Q D 2 \geq 30$ Gy $(p=0.029)$ and cumulative EQD2 $\geq 90 G y$ $(p=0.023)$ were significantly associated with better survival; frontal location ( $p=0.119)$ and KPS 80-100\% $(p=0.067)$ showed trends. In multivariate analyses, frontal location $(p=0.032)$ and cumulative $E Q D 2 \geq 90 \mathrm{~Gy}(p=0.038)$ were significant; KPS 80$100 \%(p=0.110)$ and $E Q D 2 \geq 30 \mathrm{~Gy}(p=0.083)$ showed trends. Conclusion: Predictors of survival after re-irradiation for recurrent GBM were identified that can help when designing personalized treatments. Use of irradiation with EQD2 $\geq 30 \mathrm{~Gy}$ appeared superior to lower doses.
\end{abstract}

Of all primary brain tumors in adults, glioblastoma multiforme (GBM) is the most common, with an incidence of approximately three patients per 100,000 inhabitants worldwide $(1,2)$. The prognoses of most patients with GBM are poor (3). In a randomized trial investigating a tri-modality treatment approach including maximum possible resection of the GBM followed by radio-chemotherapy over 6 weeks with concurrent

This article is freely accessible online.

Correspondence to: Professor Dirk Rades, MD, Department of Radiation Oncology, University of Lübeck, Lübeck, Ratzeburger Allee 160, 23562 Lübeck, Germany. Tel: +49 45150045401, Fax: +49 45150045404, e-mail: dirk.rades@uksh.de

Key Words: Recurrent glioblastoma, re-irradiation, survival, prognostic factors. temozolomide and additional cycles of temozolomide, median survival and 5-year survival were less than 15 months and less than $10 \%$, respectively $(4,5)$. Moreover, the median time to progression of GBM was only 6.9 months (4). The majority of recurrences of GBM occur in previously irradiated areas of the brain (in-field recurrences) and many patients receive a second course of radiotherapy (re-irradiation) (2).

The prognoses of patients re-irradiated for recurrent GBM are often poor, and treatment is palliative. In a systematic review and meta-analysis, the 1-year survival rate was only $36 \%$ after re-irradiation (2). However, selected patients may benefit from more aggressive treatment programs including resection of the recurrent GBM and systemic treatment (611). Thus, patients with recurrent GBM would likely benefit from individualized treatment taking into account various factors associated with the patient's survival prognosis in order to avoid too aggressive treatment for those with a very short expected survival and a too limited one for longer-term survivors. In general, patients with an estimated short survival time should receive a short and minimally burdensome treatment such as short-course radiotherapy (12), whereas patients with more favorable prognoses could be candidates for a more aggressive approach including reresection and administration of systemic therapies (6-11).

The present study aimed to identify predictors of survival in a cohort of patients receiving re-irradiation for recurrent GBM and contribute to treatment personalization for this particular group of patients. Moreover, it aimed to contribute to the definition of the optimal dose of re-irradiation

\section{Patients and Methods}

Twenty-six patients irradiated for recurrent GBM (re-irradiation) between 2005 and 2018 were included in this retrospective study, which was approved by the local Ethics Committee (University of Lübeck, reference number 15-355A). Total doses ranged between 15.0 and $60.0 \mathrm{~Gy}$ (median=30.0 Gy). Doses per fraction were 1.21.5 Gy (twice daily) or 1.8-7.0 Gy (once daily). Radiation techniques included volumetric-modulated arc therapy in 12 , fractionated stereotactic radiotherapy in 10 and 3D-conformal radiotherapy in 6 patients. All patients received additional systemic treatment prior to, 
Table I. Characteristics included in the analyses of survival after a second course of radiotherapy (RT) for recurrent glioblastoma (reirradiation).

\begin{tabular}{lr}
\hline Factor & No. of patients $(\%)$ \\
\hline Main site of recurrent GBM & \\
Frontal & $6(21.4)$ \\
Temporal & $11(39.3)$ \\
Parietal/occipital & $7(25.0)$ \\
Central & $4(14.3)$ \\
Type of recurrence & \\
In-field only & $21(75.0)$ \\
Out-field with/without in-field & $7(25.0)$ \\
Karnofsky performance score & \\
$50-70 \%$ & $15(53.6)$ \\
$80-100 \%$ & $13(46.4)$ \\
Gender & $11(39.3)$ \\
Female & $17(60.7)$ \\
Male & \\
Age at RT of recurrence & $15(53.6)$ \\
$\leq 60$ Years & $13(46.4)$ \\
$>60$ Years & \\
Interval between primary RT and recurrence & $15(53.6)$ \\
$\leq 12$ Months & $13(46.4)$ \\
$>12$ Months & \\
Gross total resection & $22(78.6)$ \\
No & $6(21.4)$ \\
Yes & \\
EQD2 of re-irradiation (re-RT) & $9(32.1)$ \\
$\leq 30$ Gy & $19(67.9)$ \\
$>30$ Gy & $12(42.9)$ \\
Cumulative EQD2 of primary RT plus re-RT & $16(57.1)$ \\
$>90$ Gy & \\
\hline
\end{tabular}

GBM: Glioblastoma multiforme; EQD2: equivalent dose in 2-Gy fractions.

during and/or following re-irradiation with temozolomide alone in 16 , temozolomide and procarbazine/lomustine (PC) or carmustine in six, temozolomide and bevacizumab in two, procarbazine/lomustine alone in two, bevacizumab alone in one and procarbazine/lomustine PC plus bevacizumab in one patient. Resection of recurrent GBM was performed in eight patients $(28.6 \%)$; in six of these, gross total resection (GTR) was achieved.

Nine potential prognostic factors were analyzed with respect to survival. These factors were: i) Main site of the recurrent GBM (frontal $v s$. temporal $v s$. parietal and/or occipital $v s$. central), ii) type of recurrence (in-field only $v s$. out-field with/without in-field), iii) Karnofsky performance score (KPS) at the time of re-irradiation $(50-70 \%$ vs. $80-100 \%$, median $=70 \%)$, iv) gender, v) age at reirradiation $(\leq 60$ vs. $>60$ years, median $=60$ years $)$, vi) interval between end of primary radiotherapy of GBM and recurrence $(\leq 12$ $v s .>12$ months, median interval=12 months), vii) GTR of recurrent GBM (no vs. yes), viii) equivalent dose in 2-Gy fractions (EQD2) of re-irradiation ( $<30 \mathrm{~Gy} v s . \geq 30 \mathrm{~Gy}$ ), ix) and cumulative EQD2 of the primary radiotherapy and re-irradiation ( $<90 \mathrm{~Gy} v s . \geq 90 \mathrm{~Gy})$ $(13,14)$. Distributions of the factors are summarized in Table I.

Survival was calculated from the first day of re-irradiation. Univariate analyses were performed applying the Kaplan-Meier
Table II. Survival rates (univariate analyses) at 6 and 12 months after start of radiotherapy $(R T)$ for recurrent glioblastoma (re-irradiation).

\begin{tabular}{|c|c|c|c|}
\hline Factor & $\begin{array}{l}6 \text { Months } \\
(\%)\end{array}$ & $\begin{array}{l}12 \text { Months } \\
(\%)\end{array}$ & $p$-Value \\
\hline \multicolumn{4}{|l|}{ Main site of recurrent GBM } \\
\hline Frontal & 83 & 83 & \multirow[t]{4}{*}{0.119} \\
\hline Temporal & 82 & 18 & \\
\hline Parietal/occipital & 86 & 29 & \\
\hline Central & 25 & 0 & \\
\hline \multicolumn{4}{|l|}{ Type of recurrence } \\
\hline In-field only & 76 & 33 & \multirow[t]{2}{*}{$>0.99$} \\
\hline Out-field with/without in-field & 71 & 29 & \\
\hline \multicolumn{4}{|l|}{ Karnofsky performance score } \\
\hline $50-70 \%$ & 67 & 13 & \multirow[t]{2}{*}{0.067} \\
\hline $80-100 \%$ & 85 & 54 & \\
\hline \multicolumn{4}{|l|}{ Gender } \\
\hline Female & 91 & 27 & \multirow[t]{2}{*}{0.62} \\
\hline Male & 65 & 35 & \\
\hline \multicolumn{4}{|l|}{ Age at RT of recurrence } \\
\hline$\leq 60$ Years & 80 & 46 & \multirow{2}{*}{0.16} \\
\hline$>60$ Years & 69 & 15 & \\
\hline \multicolumn{4}{|l|}{ Interval between primary } \\
\hline \multicolumn{4}{|l|}{ RT and recurrence } \\
\hline$\leq 12$ Months & 60 & 27 & \multirow[t]{2}{*}{0.30} \\
\hline$>12$ Months & 92 & 38 & \\
\hline \multicolumn{4}{|l|}{ Gross total resection } \\
\hline No & 68 & 22 & \multirow[t]{2}{*}{0.047} \\
\hline Yes & 100 & 67 & \\
\hline \multicolumn{4}{|l|}{ EQD2 of re-irradiation (re-RT) } \\
\hline$<30 \mathrm{~Gy}$ & 44 & 11 & \multirow[t]{2}{*}{0.029} \\
\hline$\geq 30 \mathrm{~Gy}$ & 89 & 42 & \\
\hline \multicolumn{4}{|l|}{$\begin{array}{l}\text { Cumulative EQD2 of } \\
\text { primary RT plus re-RT }\end{array}$} \\
\hline$<90 \mathrm{~Gy}$ & 50 & 8 & \multirow[t]{3}{*}{0.023} \\
\hline$\geq 90 \mathrm{~Gy}$ & 94 & 50 & \\
\hline Entire cohort & 75 & 32 & \\
\hline
\end{tabular}

GBM: Glioblastoma multiforme; EQD2: equivalent dose in 2-Gy fractions. Statistically significant $p$-values are shown in bold.

method and the Wilcoxon test. $p$-Values less than 0.05 were regarded as significant, and $p$-values $<0.12$ as indicating a trend. The significant factors were subsequently included in a multivariate analysis (Cox regression model).

\section{Results}

Median follow-up for the entire cohort was 9 months (range $=2-34$ months). Twenty-four patients $(85.7 \%)$ died during the follow-up after median of 9 months. Median follow-up for the remaining four patients was 11 months (range $=9-15$ months). For the entire cohort, the survival rates at 6 months and 12 months were $75 \%$ and $32 \%$, respectively. On univariate analyses (Table II), GTR ( $p=0.047)$, an EQD2 of re-irradiation $\geq 30$ Gy $(p=0.029$, Figure 1$)$ and a cumulative EQD2 of primary and re-irradiation $\geq 90$ Gy $(p=0.023$, Figure 


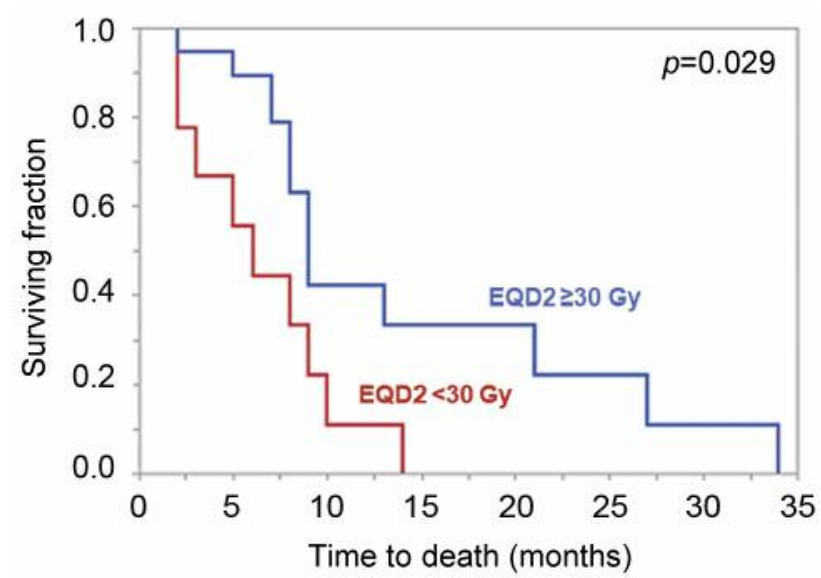

Figure 1. Kaplan-Meier curves (univariate analysis) for the survival of patients receiving re-irradiation with an equivalent dose in 2-Gy fractions (EQD2) of $\geq 30$ Gy vs. $<30 \mathrm{~Gy}$ ).

2) were significantly associated with more favorable survival. In addition, trends were found for frontal location of GBM $(p=0.119)$ and a KPS of $80-100 \%(p=0.067)$.

In the subsequent multivariate analyses, frontal location [risk ratio $(\mathrm{RR})=1.65,95 \%$ confidence interval $(\mathrm{CI})=1.04$ 2.68, $p=0.032]$ and cumulative EQD2 $\geq 90$ Gy $(\mathrm{RR}=2.57$, $95 \% \mathrm{CI}=1.05-6.49, p=0.038)$ were significantly associated with more favorable survival. KPS of $80-100 \%$ ( $R R=2.24$, 95\% CI=0.84-6.21, $p=0.110)$ and $\mathrm{EQD} 2 \geq 30 \mathrm{~Gy}(\mathrm{RR}=2.27$, $95 \% \mathrm{CI}=0.89-5.52, p=0.083)$ showed trends, and GTR $(\mathrm{RR}=2.17,95 \% \mathrm{CI}=0.66-9.71, p=0.21)$ was not significant.

\section{Discussion}

GBM is the most common primary brain tumor in adults and is often associated with a poor prognosis (1-3). Despite the introduction of multi-modality treatment approaches and considerable pre-clinical and clinical research, the prognoses of patients with GBM need to be improved (4, 5, 15-17). The poor prognoses are mainly the consequence of intracerebral recurrences. In the trial of Stupp et al., median progressionfree survival was less than 7 months after tri-modality treatment with neurosurgical resection followed by concurrent radiochemotherapy (60 Gy in 30 fractions over 6 weeks plus temozolomide) and maintenance treatment with temozolomide alone $(4,5)$.

When experiencing a recurrence of GBM, many patients receive re-irradiation. For this situation, the optimal dose of re-irradiation needs further clarification. Since many different dose-fractionation regimens are used for re-irradiation of recurrent GBM, the doses are often given as EQD2 in order to ensure comparability of different regimens $(13,14)$. The EQD2 considers both the total dose and dose per fraction and

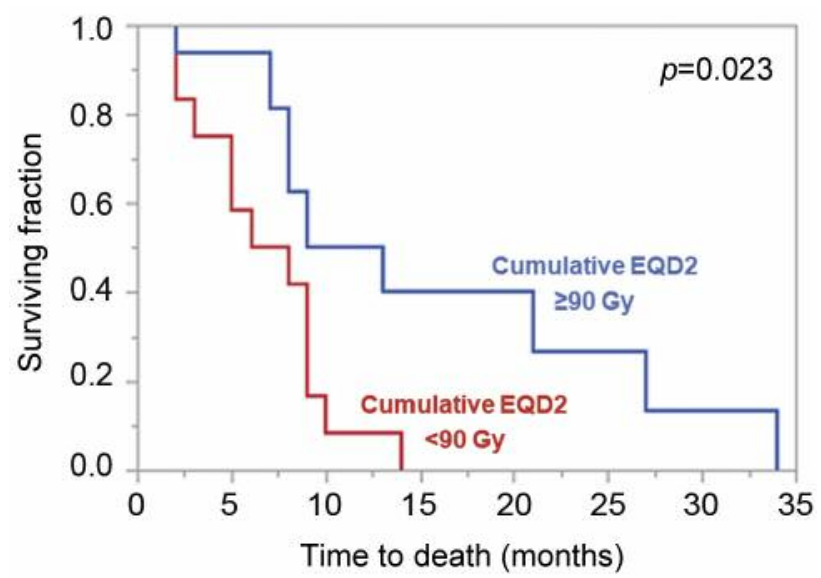

Figure 2. Kaplan-Meier curves (univariate analysis) for the survival of patients receiving a cumulative equivalent dose in 2-Gy fractions (EQD2) of primary radiotherapy and re-irradiation of $\geq 90$ Gy vs. $<90 \mathrm{~Gy}$.

is based on the linear-quadratic model. The alpha/beta ratio represents the dose, where cell killing from the linear and the quadratic components are equal. The alpha/beta ratio for tumor cell kill is considered to be $10 \mathrm{~Gy}$ for the vast majority of malignant tumors. In a previous meta-analysis, no significant difference regarding outcomes after re-irradiation (external beam radiation therapy) of recurrent GBM were found between EQD2 <36 Gy and $\geq 36$ Gy (2). However, the authors stated that in several studies included in their metaanalysis, many different dose-fractionation regimens were administered and that using the median EQD2 may not be the optimal method for defining a dose-effect relationship (2). A dose-effect relationship was found in a retrospective study of 20 patients with malignant gliomas (19 with GBM) receiving hypo-fractionated stereotactic radiotherapy (HFSRT) for persistent or recurrent disease (18). Response rates were $0 \%$ after $24 \mathrm{~Gy}$ in eight fractions (EQD2=26.0 Gy) and 79\% after 30 Gy or 35 Gy in 10 fractions (EQD2=32.5 Gy or 39.4 Gy), respectively. In another retrospective study of 19 patients (14 with GBM, five with anaplastic astrocytoma) treated with HFSRT for recurrent disease, absolute total doses (not EQD2) of $\geq 30$ Gy resulted in a borderline significantly better median survival than doses $<30$ Gy (11.1 vs. 7.4 months, $p=0.051$ ) (19). Since findings regarding the optimal dose of reirradiation conflict, additional studies would help better define the optimal dose for recurrent GBM. Moreover, additional cutoff doses of the EQD2 should be investigated.

The present study compared EQD2 doses of $<30$ Gy to $\geq 30$ Gy. On univariate analysis, doses $\geq 30$ Gy were significantly superior to $<30 \mathrm{~Gy}$, resulting in an absolute difference of $45 \%$ points $(89 \%$ vs. $44 \%)$ at 6 months and $31 \%$ points $(42 \%$ vs. $11 \%$ ) at 12 months. In the multivariate analysis, the results showed at least a trend. In addition, the cumulative EQD2 of 
the radiotherapy of the primary GBM and re-irradiation of the recurrent GBM was significantly associated with survival in both univariate and multivariate analyses; doses $\geq 90$ Gy were significantly superior to $<90 \mathrm{~Gy}$.

In addition to the appropriate dose of re-irradiation, other pre-radiotherapy factors showed a significant association with survival or at least a trend. Frontal location of recurrent GBM was significantly associated with better survival in the multivariate analysis, demonstrating the main site of GBM to be an independent predictor of survival. A better performance status (KPS of 80-100\%) showed a trend for being associated with better survival in both univariate and multivariate analyses, and GTR was significantly associated with improved survival on univariate analysis. These prognostic factors can be used to support physicians who aim to select a personalized treatment for a patient with recurrent GBM. Patients with one or more of these favorable prognostic factors may benefit from longer-course radiotherapy with higher doses that may be combined with systemic treatment (6). Patients without favorable prognostic factors appear more appropriately treated with short-course radiotherapy alone such as $5 \times 5$ Gy given over 1 week (12).

When considering these suggestions, one should keep in mind the limitations of the present study. These include the limited size and retrospective nature of the data always bearing the risk of a hidden selection bias. However, the 6-and 12 -month survival rates of this study (75\% and $32 \%)$ were similar to those reported in the meta-analysis of Kazmi et al. (73\% and $36 \%$ ) and within the $95 \%$ confidence intervals (69$77 \%$ and $32-40 \%$ ) of that meta-analysis (2). This demonstrates consistency of the data of our present study. The consistency is further supported by the fact that the three pre-radiotherapy predictors of survival were found in other studies. In 2007, Carson et al. presented data of 333 patients with recurrent glioma and found positive associations of survival with KPS $\geq 80 \%$ and with tumors confined to the frontal lobe (20). Similar results were reported in a pooled analysis of 300 patients with recurrent GBM from eight phase I or II trials (21). KPS $\geq 80 \%$ was significantly associated with improved survival in two studies and a pooled analysis from Germany including patients with recurrent gliomas of any grade (22-24). The value of re-resection has been shown in several studies (7-11). Two of these studies performed comparisons of reirradiation with $v s$. without re-resection $(8,11)$. In the retrospective study of Skeie et al., median survival times were 9 months after Gamma Knife radiosurgery alone $(n=32)$ and 15 months after Gamma Knife radiosurgery plus re-resection $(n=19)$ (11). In the retrospective study of Kim et al. that included 36 patients with recurrent GBM, surgical resection when added to stereotactic radiosurgery or HFSRT was associated with significantly improved survival $(p=0.010)$, and the extent of resection showed a trend for being positively associated with survival $(p=0.071)(8)$.
In summary, predictors of survival after re-irradiation for recurrent GBM were identified that can help when designing personalized treatments and future clinical trials. Use of an EQD2 of re-irradiation $\geq 30$ Gy and a cumulative EQD2 of primary and re-irradiation $\geq 90 \mathrm{~Gy}$ appeared superior to lower doses and should be administered.

\section{Conflicts of Interest}

The Authors report no conflicts of interest related to the present study.

\section{Authors' Contributions}

The study was designed by all Authors. The data were collected by J.W. and analyzed by D.R. and S.E.S. The article was drafted by D.R. and S.E.S. and finally approved by all Authors.

\section{References}

1 Louis DN, Perry A, Reifenberger G, von Deimling A, FigarellaBranger D, Cavenee WK, Ohgaki H, Wiestler OD, Kleihues P and Ellison DW: The 2016 World Health Organization Classification of Tumors of the Central Nervous System: A summary. Acta Neuropathol 131: 803-820, 2016. PMID: 27157931. DOI: $10.1007 / \mathrm{s} 00401-016-1545-1$

2 Kazmi F, Soon YY, Leong YH, Koh WY and Vellayappan B: Reirradiation for recurrent glioblastoma (GBM): A systematic review and meta-analysis. J Neurooncol 142: 79-90, 2019. PMID: 30523605. DOI: 10.1007/s11060-018-03064-0

3 Gittleman H, Boscia A, Ostrom QT, Truitt G, Fritz Y, Kruchko $\mathrm{C}$ and Barnholtz-Sloan JS: Survivorship in adults with malignant brain and other central nervous system tumor from 2000-2014. Neuro Oncol 20(suppl 7): vii6-vii16, 2018. PMID: 29850889. DOI: 10.1093/neuonc/noy090.

4 Stupp R, Mason WP, van den Bent MJ, Weller M, Fisher B, Taphoorn MJ, Belanger K, Brandes AA, Marosi C, Bogdahn U, Curschmann J, Janzer RC, Ludwin SK, Gorlia T, Allgeier A, Lacombe D, Cairncross JG, Eisenhauer E and Mirimanoff RO; European Organisation for Research and Treatment of Cancer Brain Tumor and Radiotherapy Groups; National Cancer Institute of Canada Clinical Trials Group: Radiotherapy plus concomitant and adjuvant temozolomide for glioblastoma. N Engl J Med 352: 987-996, 2005. PMID: 15758009. DOI: 10.1056/NEJMoa043330

5 Stupp R, Hegi ME, Mason WP, van den Bent MJ, Taphoorn MJ, Janzer RC, Ludwin SK, Allgeier A, Fisher B, Belanger K, Hau P, Brandes AA, Gijtenbeek J, Marosi C, Vecht CJ, Mokhtari K, Wesseling P, Villa S, Eisenhauer E, Gorlia T, Weller M, Lacombe D, Cairncross JG and Mirimanoff RO; European Organisation for Research and Treatment of Cancer Brain Tumour and Radiation Oncology Groups; National Cancer Institute of Canada Clinical Trials Group: Effects of radiotherapy with concomitant and adjuvant temozolomide versus radiotherapy alone on survival in glioblastoma in a randomised phase III study: 5-Year analysis of the EORTC-NCIC trial. Lancet Oncol 10: 459-466, 2009. PMID: 19269895. DOI: 10.1016/S1470-2045(09)70025-7

6 Shi W, Scannell Bryan M, Gilbert MR, Mehta MP, Blumenthal DT, Brown PD, Valeinis E, Hopkins K, Souhami L, Andrews DW, 
Tzuk-Shina T, Howard SP, Youssef EF, Lessard N, Dignam JJ and Werner-Wasik M: Investigating the Effect of reirradiation or systemic therapy in patients with glioblastoma after tumor progression: A secondary analysis of NRG Oncology/Radiation Therapy Oncology Group trial 0525. Int J Radiat Oncol Biol Phys 100: 38-44, 2018. PMID: 29102648. DOI: 10.1016/j.ijrobp. 2017.08.038

7 van Linde ME, Brahm CG, de Witt Hamer PC, Reijneveld JC, Bruynzeel AME, Vandertop WP, van de Ven PM, Wagemakers M, van der Weide HL, Enting RH, Walenkamp AME and Verheul HMW: Treatment outcome of patients with recurrent glioblastoma multiforme: A retrospective multicenter analysis. J Neurooncol 135: 183-192, 2017. PMID: 28730289. DOI: 10.1007/s11060-017-2564-Z

8 Kim MS, Lim J, Shin HS and Cho KG: Re-Irradiation and its contribution to good prognosis in recurrent glioblastoma patients. Brain Tumor Res Treat 8: 29-35, 2020. PMID: 32390351. DOI: $10.14791 /$ btrt.2020.8.e10

9 Azoulay M, Santos F, Shenouda G, Petrecca K, Oweida A, Guiot MC, Owen S, Panet-Raymond V, Souhami L and Abdulkarim BS: Benefit of re-operation and salvage therapies for recurrent glioblastoma multiforme: results from a single institution. J Neurooncol 132: 419-426, 2017. PMID: 28374095. DOI: 10.1007/s11060-017-2383-2

10 Wann A, Tully PA, Barnes EH, Lwin Z, Jeffree R, Drummond $\mathrm{KJ}$, Gan $\mathrm{H}$ and Khasraw M: Outcomes after second surgery for recurrent glioblastoma: A retrospective case-control study. J Neurooncol 137: 409-415, 2018. PMID: 29294233. DOI: $10.1007 / \mathrm{s} 11060-017-2731-2$

11 Skeie BS, Enger PØ, Brøgger J, Ganz JC, Thorsen F, Heggdal JI and Pedersen PH: $\gamma$ Knife surgery versus reoperation for recurrent glioblastoma multiforme. World Neurosurg 78: 658669, 2012. PMID: 22484078. DOI: 10.1016/j.wneu.2012.03.024

12 Roa W, Kepka L, Kumar N, Sinaika V, Matiello J, Lomidze D, Hentati D, Guedes de Castro D, Dyttus-Cebulok K, Drodge S, Ghosh S, Jeremić B, Rosenblatt E and Fidarova E: International Atomic Energy Agency randomized phase III study of radiation therapy in elderly and/or frail patients with newly diagnosed glioblastoma multiforme. J Clin Oncol 33: 4145-4150, 2015. PMID: 26392096. DOI: 10.1200/JCO.2015.62.6606

13 Barendsen GW: Dose fractionation, dose rate and iso-effect relationships for normal tissue responses. Int J Radiat Oncol Biol Phys 8: 1981-1997, 1982. PMID: 6759484. DOI: 10.1016/03603016(82)90459-x

14 Joiner MC and Van der Kogel AJ: The linear-quadratic approach to fractionation and calculation of isoeffect relationships. In: Basic Clinical Radiobiology. Steel GG (ed.). New York, Oxford University Press, pp. 106-112, 1997.

15 Antonopoulos M, van Gool SW, Dionysiou D, Graf N and Stamatakos G: Immune phenotype correlates with survival in patients with GBM treated with standard temozolomide-based therapy and immunotherapy. Anticancer Res 39: 2043-2051, 2019. PMID: 30952748. DOI: 10.21873/anticanres.13315

16 Romanenko MV, Dolgova EV, Osipov ID, Ritter GS, Sizova MS, Proskurina AS, Efremov YR, Bayborodin SI, Potter EA, Taranov OS, Omigov VV, Kochneva GV, Grazhdantseva AA, Zavyalov EL, Razumov IA, Netesov SV and Bogachev SS: Oncolytic effect of adenoviruses serotypes 5 and 6 against U87 glioblastoma cancer stem cells. Anticancer Res 39: 6073-6086, 2019. PMID: 31704835. DOI: 10.21873/anticanres.13815
17 Toda Y, Yoshimura R, Itahara M, Imai Y, Yamada K, Uno T, Nakata S, Hosogi S, Takata K and Ashihara E: DJ-1 Contributes to self-renewal of stem cells in the U87-MG glioblastoma cell line. Anticancer Res 39: 5983-5990, 2019. PMID: 31704823. DOI: 10.21873 /anticanres.13803

18 Hudes RS, Corn BW, Werner-Wasik M, Andrews D, Rosenstock J, Thoron L, Downes B and Curran WJ Jr: A phase I dose escalation study of hypofractionated stereotactic radiotherapy as salvage therapy for persistent or recurrent malignant glioma. Int J Radiat Oncol Biol Phys 43: 293-298, 1999. PMID: 10030252. DOI: $10.1016 / \mathrm{s} 0360-3016(98) 00416-7$

19 Vordermark D, Kölbl O, Ruprecht K, Vince GH, Bratengeier K and Flentje $\mathrm{M}$ : Hypofractionated stereotactic re-irradiation: Treatment option in recurrent malignant glioma. BMC Cancer 5: 55, 2005. PMID: 15924621. DOI: 10.1186/1471-2407-5-55

20 Carson KA, Grossman SA, Fisher JD and Shaw EG: Prognostic factors for survival in adult patients with recurrent glioma enrolled onto the new approaches to brain tumor therapy CNS consortium phase I and II clinical trials. J Clin Oncol 25: 26012606, 2007. PMID: 17577040 . DOI: $10.1200 / J C O .2006 .08 .1661$

21 Gorlia T, Stupp R, Brandes AA, Rampling RR, Fumoleau P, Dittrich C, Campone MM, Twelves CC, Raymond E, Hegi ME, Lacombe D and van den Bent MJ: New prognostic factors and calculators for outcome prediction in patients with recurrent glioblastoma: A pooled analysis of EORTC Brain Tumour Group phase I and II clinical trials. Eur J Cancer 48: 1176-1184, 2012. PMID: 22464345. DOI: 10.1016/j.ejca.2012.02.004

22 Kessel KA, Hesse J, Straube C, Zimmer C, Schmidt-Graf F, Schlegel J, Meyer B and Combs SE: Validation of an established prognostic score after re-irradiation of recurrent glioma. Acta Oncol 56: 422-426, 2017. PMID: 28075197. DOI: 10.1080/0284 186X.2016.1276621

23 Kessel KA, Hesse J, Straube C, Zimmer C, Schmidt-Graf F, Schlegel J, Meyer B and Combs SE: Modification and optimization of an established prognostic score after reirradiation of recurrent glioma. PLoS One 12: e0180457, 2017. PMID: 28678889. DOI: 10.1371/journal.pone.0180457

24 Combs SE, Niyazi M, Adeberg S, Bougatf N, Kaul D, Fleischmann DF, Gruen A, Fokas E, Rödel CM, Eckert F, Paulsen F, Oehlke O, Grosu AL, Seidlitz A, Lattermann A, Krause M, Baumann M, Guberina M, Stuschke M, Budach V, Belka C, Debus J and Kessel KA: Re-irradiation of recurrent gliomas: pooled analysis and validation of an established prognostic score-report of the Radiation Oncology Group (ROG) of the German Cancer Consortium (DKTK). Cancer Med 7: 1742-1749, 2018. PMID: 29573214. DOI: 10.1002/cam4.1425

Received October 27, 2020

Revised November 2, 2020

Accepted November 3, 2020 